\title{
Polynomial Load Model Development for Analysing Residential Electric Energy Use Behaviour
}

\author{
Erwin Nashrullah ${ }^{1}$, Abdul Halim ${ }^{1, *}$ \\ ${ }^{1}$ Department of Electrical Engineering, Faculty of Engineering, Universitas Indonesia
}

\begin{abstract}
Analysing and simulating the dynamic behaviour of home power system as a part of community-based energy system needs load model of either aggregate or dis-aggregate power use. Moreover, in the context of home energy efficiency, development of specific and accurate residential load model can help system designer to develop a tool for reducing energy consumption effectively. In this paper, a new method for developing two types of residential polynomial load model is presented. In the research, computation technique of model parameters is provided based on median filter and least square estimation and implemented by MATLAB. We use AMPDs data set, which have 1-minute data sampling, to show the effectiveness of proposed method. After simulation is carried out, the performance evaluation of model is provided through exploring root mean-squared error between original data and model output. From simulation results, it could be concluded that proposed model is enough for helping system designer to analyse home power energy use.
\end{abstract}

\section{Introduction}

Community-based energy system is becoming promising scheme to overcome some fundamental human energy issues such as operational efficiency and local resource utilization. The system allows the participation and ownership of citizens or members of a defined community in operational aspect. The characteristic of the system is formed by at least two of the following elements: 1) Most of a renewable energy project is owned by local stakeholders, 2) Community institution takes the control, 3) Most of social and economic benefits are distributed locally [1]. In this paper, we limit the definition of community-based energy system as electric power system owned and operated by community.

Community-based energy system adopts smart micro grid architecture. Microgrid means a power delivery infrastructure in micro size $(<1 \mathrm{MW})$ which has small-scale generating units, energy storage, and loads. Microgrid normally operates connected to a traditional centralised grid and has capability in self-islanding operation. Meanwhile smart grid means that the system accommodates information communication technologies

\footnotetext{
Corresponding author: a.halim@ui.ac.id
} 
actively to connect consumers and producer's needs. Smart grid makes the system operation efficient due to sharing information $[2,3]$.

To design the system, the simulation tool is required. The simulation is also needed for performance evaluation. In order to develop a simulator, mathematical model of system must be derived firstly. In this paper, we present mathematical models of home electrical load. The load changes continuously due to the operation of home appliances and other equipment. The models proposed here are called as multiplicative and additive polynomial model. The models derived are static non-linear and derived by using least square method. Furthermore, Almanac of Minutely Power dataset (AMPDs) is used as case study [4]. Median filter is utilized to eliminate the noise. In this research the performance of median filter is also evaluated. By using MATLAB, the calculation of model parameters is conducted, and models are validated.

\section{Mathematical Model}

\subsection{Residential Load Data}

Smart meters have become mandatory for smart grid. The use of digital smart meter effects to the volume of information acquired and its quality. By using this tool, the consumption patterns of different appliances inside the house can be grasped in more accurate. The insight into the residential load consumption has advantages for both the energy provider as well as for the consumer such for managing energy efficiently [7].

Residential load data can be classified as low sampling and high sampling. High sampling rate $\geq 50 \mathrm{~Hz}(\leq 0.02 \mathrm{~s}$ ) (up to several $\mathrm{kHz}$ ). While low sampling rate $\leq 1 \mathrm{~Hz}(\geq 1 \mathrm{~s})$. AMPDs has 1-minute sampling rate so that it enters the class of low sampling rate. AMPDs dataset includes voltage, frequency, active and reactive power. In each dataset, The emergence of spike and noise cause the model derivation more difficult.

\subsection{Median Filter}

Median filter is a nonlinear mathematical tool for repressing noise while retaining sharp sustained changes (edges) in signal values. The filter is particularly effective in reducing impulsive-type noise. The filter outputs median value of the input data inside the window centered at the point [6]. Median filter can be defined as follows [7]:

For a set of $m$ numbers $\left\{z_{1}, z_{2}, \cdots\right.$, if they are arranged in order such that $z_{1} \leq z_{2} \leq \ldots \quad \ldots$.The median of $m$ numbers $\left\{z_{1}, z_{2}, \ldots \ldots\right.$ is the $(\xi+1)$ th-order statistic, where $\xi=(m-1) / 2$ if $m$ is odd, and if $m$ is even. In this paper, set of $m$ numbers is called n-window.

\subsection{Polynomial Load Models}

Polynomial load model is commonly used in analysis and design of power system. ZIP model is a model type classified as polynomial model [8,9]. Different to model ZIP model, in this paper we propose two type models that are called as Multiplicative and Additive Polynomial Model. The mathematical models are:

a. n- Order Multiplicative Polynomial Model 


$$
\begin{aligned}
& \left.P_{l}(V)=P_{l o}\left[a_{n}\left(\frac{V}{V_{o}}\right)^{n}+\cdots \quad \begin{array}{l}
\left(V_{-}^{-}\right. \\
V_{o}^{-}
\end{array}\right)^{2}+a_{1}\left(\frac{V}{V_{o}}\right)+a_{0}\right] \\
& Q_{l}(V)=Q_{l o}\left[b_{n}\left(\frac{V}{V_{o}}\right)^{n}+\cdots \quad\left(\begin{array}{c}
V \\
V_{o}^{-}
\end{array}\right)^{2}+b_{1}\left(\frac{V}{V_{o}}\right)+b_{0}\right]
\end{aligned}
$$

b. n- Order Additive Polynomial Model

$$
\begin{aligned}
& P_{l}(V)=P_{l}\left(V_{o}\right)\left[\alpha_{o}+\alpha_{1} \frac{\left(V-V_{o}\right)}{V_{o}}+\cdots \quad \cdots \frac{\left(V-V_{o}\right)^{n}}{V_{o}^{n}}\right] \\
& Q_{l}(V)=Q_{l}\left(V_{o}\right)\left[\beta_{o}+\beta_{1} \frac{\left(V-V_{o}\right)}{V_{o}}+\cdots \quad \frac{\left(V-V_{o}\right)^{n}}{V_{o}^{n}}\right]
\end{aligned}
$$

where $V_{o}$ : rated voltage; $V$ : actual operating voltage, $P_{l}, Q_{l}$ : active and reactive load powers at operating voltage $; P_{l o}, Q_{l o}$ : active and reactive powers at rated voltage, $a_{0}$, $a_{1}, \ldots, a_{n}, b_{0}, b_{1}, \ldots, b_{n}, \alpha_{o}, \ldots, \alpha_{n}, \beta_{o}, \ldots, \beta_{n}$ are constant.

\subsection{Parameter Estimation Method}

Least square method is used to estimate the parameters of models (1)-(4). In the next formulas, instead using n order polynomial, for clarity and simplicity, 2-order polynomial is used. The formula to estimate the parameters is

$$
\bar{\theta}=\left(\bar{\Omega}^{\mathrm{T}} \overline{\boldsymbol{\Omega}}\right)^{-1} \overline{\boldsymbol{\Omega}}^{\mathrm{T}} \bar{\vartheta}
$$

where, for multiplicative polynomial model,

$$
\begin{aligned}
& ; \overline{\boldsymbol{\theta}}=\left[\begin{array}{llllll}
a_{2} & a_{1} & a_{o} & b_{2} & b_{1} & b_{o}
\end{array}\right]^{T} \overline{\boldsymbol{\vartheta}}=\left[\begin{array}{l}
\overline{\boldsymbol{\vartheta}}_{p} \\
\overline{\boldsymbol{\vartheta}}_{Q}
\end{array}\right] ; ; \overline{\boldsymbol{\vartheta}}_{P}=\frac{1}{P_{l_{o}}}\left[\begin{array}{lllll}
P_{l_{1}} & P_{l_{2}} & \ldots & \left.{ }^{\prime T}\right\lrcorner ;
\end{array} ;\right.
\end{aligned}
$$

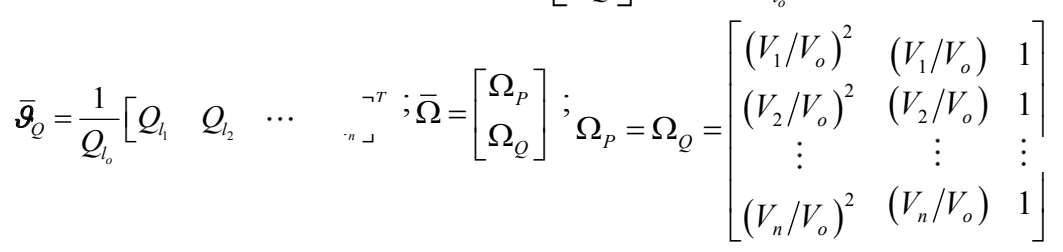

and for additive polynomial model,

$$
\begin{aligned}
& \overline{\boldsymbol{\theta}}=\left[\begin{array}{llllll}
\alpha_{o} & \alpha_{1} & \alpha_{2} & \beta_{o} & \beta_{1} & \beta_{2}
\end{array}\right]^{T} ; \overline{\boldsymbol{\vartheta}}=\left[\begin{array}{c}
\overline{\boldsymbol{\vartheta}}_{p} \\
\overline{\boldsymbol{\vartheta}}_{Q}
\end{array}\right] \overline{\boldsymbol{\vartheta}}_{P}=\frac{1}{P_{l}\left(V_{o}\right)}\left[\begin{array}{lllll}
P_{l_{1}}(V) & P_{l_{2}}(V) & \cdots & &
\end{array}\right]^{T} \\
& \bar{\vartheta}_{Q}=\frac{1}{Q_{l}\left(V_{o}\right)}\left[\begin{array}{lllll}
Q_{l_{1}}(V) & Q_{l_{2}}(V) & \cdots & & { }_{n}
\end{array}\right]^{T} ; \bar{\Omega}=\left[\begin{array}{l}
\Omega_{P} \\
\Omega_{Q}
\end{array}\right]
\end{aligned}
$$




$$
\Omega_{P}=\Omega_{Q}=\left[\begin{array}{ccc}
1 & \left(V_{1}-V_{o}\right) / V_{o} & \left(\left(V_{1}-V_{o}\right) / V_{o}\right)^{2} \\
1 & \left(V_{2}-V_{o}\right) / V_{o} & \left(\left(V_{2}-V_{o}\right) / V_{o}\right)^{2} \\
\vdots & \vdots \\
1 & \left(V_{n}-V_{o}\right) / V_{n} & \left(\left(V_{n}-V_{o}\right) / V_{o}\right)^{2}
\end{array}\right]
$$

\section{Simulation And Analysis}

In this section, we will present the performance evaluation of median filters, model parameter computation results and model validation.

\subsection{Performance Evaluation of Median Filter}

In this reseach, AMPDs data has been studied to evaluate median filter. Before processing by median filter or original data of active power, reactive power and voltage profiles are given in Figure 1. As shown in this figure, the big problem is the existance of noise in each profiles particularly in voltage profile.

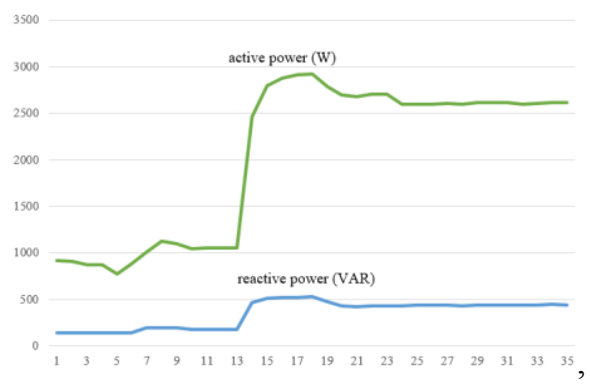

(a) Active and Reactive Power

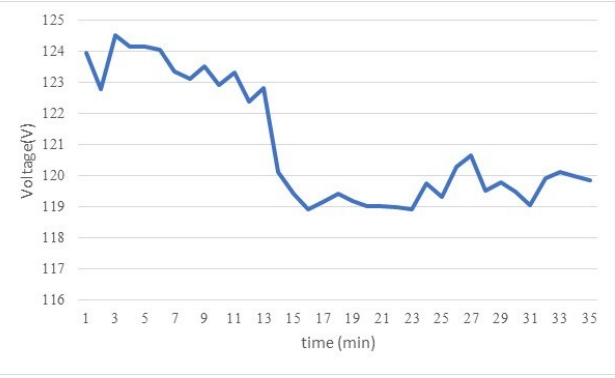

(b) Voltage

Fig. 4. Pre-processing AMPDs Data

In the next, smoothing of active power, reactive power and voltage curve are deployed by using median filter with 3,5,7 and 9-window. The results are shown in Figure 2. From these figures, it could be concluded that:

a. Median filter has ability to smooth the curve and preserve the edge values.

b. The bigger value of $n$-window results smoother curve.

c. However, bigger value of n-window potentially suffers the curve so that some characteristics of data will lost.

Based on the above analysis, in this paper, 5-window median filter is used to proceed raw data and formulate the model.

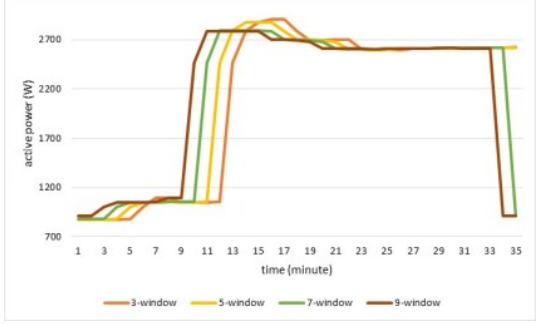

(a) Active Power

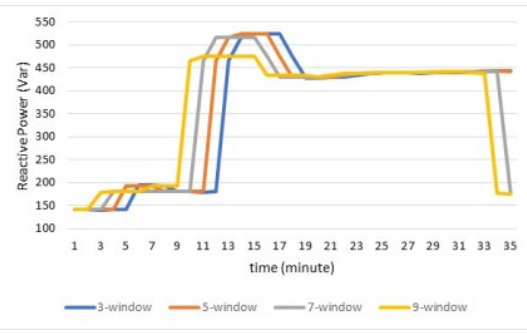

(b) Reactive Power 


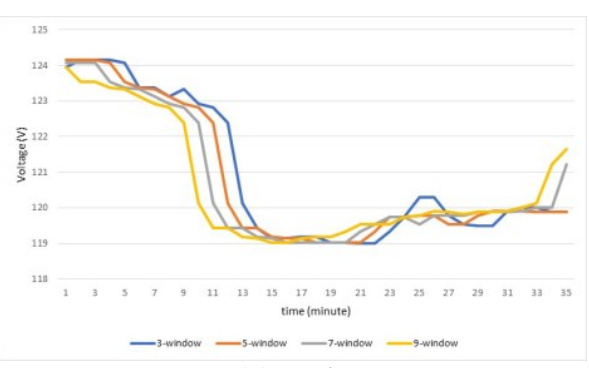

(c) Voltage

Fig. 2. Data Profiles in Four Cases of Windows

\subsection{Parameter Estimation and Validation Result}

Calculation of parameters is carried out by using MATLAB. The results are as follows. In all case, it is set $V_{o}=120 \mathrm{~V}$.

For Multiplicative Model, with $P_{l o}=3000 Q_{l o}=500$, the model is

$$
\begin{aligned}
& P_{l}(V)=P_{l o}\left[58.40\left(\frac{V}{V_{o}}\right)^{2}-134.95\left(\frac{V}{V_{o}}\right)+77.37\right] \\
& Q_{l}(V)=Q_{l o}\left[(-25.62)\left(\frac{V}{V_{o}}\right)^{2}+35.01\left(\frac{V}{V_{o}}\right)-8.55\right]
\end{aligned}
$$

RMSE (Root Mean Squared Error) is 0.0438 and 0.0675 resectively. Graphical result is depicted in Figure 3.

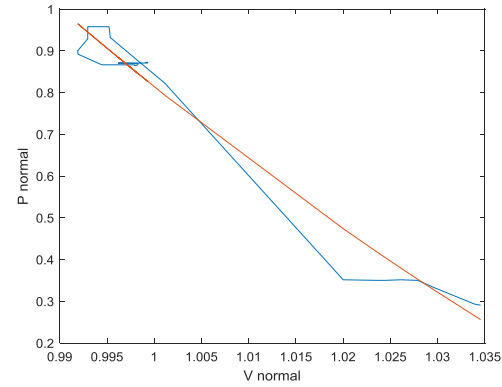

(a) Active Power

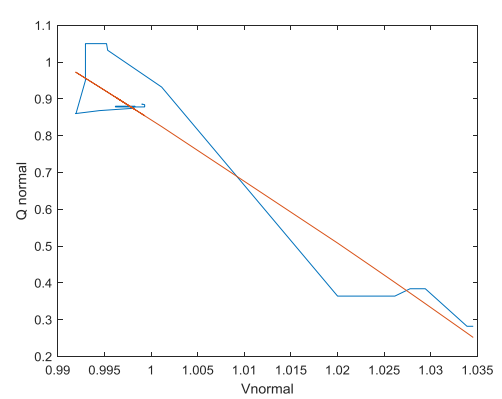

(b) Reactive Power

Fig.3. Comparison of Real vs Model (Multiplicative Type)

For Additive Model with $P\left(V_{o}\right)=3000$ and $Q\left(V_{o}\right)=500$, the model is

$$
\begin{aligned}
& P_{l}(V)=P\left(V_{o}\right)\left[0.840-18.17\left(\frac{V-V_{o}}{V_{o}}\right)+58.4\left(\frac{V-V_{o}}{V_{o}}\right)^{2}\right] \\
& Q_{l}(V)=Q_{l}\left(V_{o}\right)\left[0.840-16.07\left(\frac{V-V_{o}}{V_{o}}\right)-27.84\left(\frac{V-V_{o}}{V_{o}}\right)^{2}\right]
\end{aligned}
$$


RMSE (Root Mean Squared Error) is 0.0438 and 0.0679 resectively. Graphical result is depicted in Figure 4.

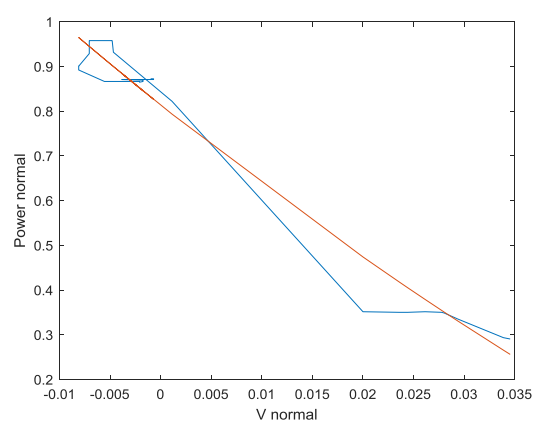

(a) Active Power

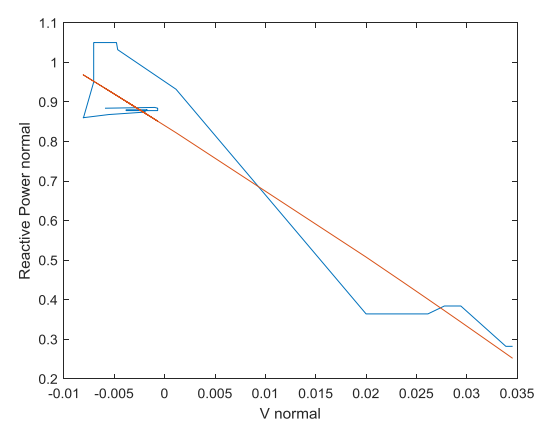

(b) Reactive Power

Fig.4. Comparison of Real vs Model (Additive Type)

\section{Conclusions}

The aim of this paper is to provide model for representing dynamic residential load. There are two models presented here which are multiplicative and additive load model. The parameters of models have been estimated by using least squared error method. Before model is derived, median filter with 5-window has been used. Using AMPDs data, it has been verified that two models have almost same performance. Moreover, some improvements can be done to reduce error between model and real data,

We acknowledge the support from Hibah PITTA 2018 funded by DRPM Universitas Indonesia No.2356/UN2.R3.1/ HKP.05.00/2018.

\section{References}

1. International Renewable Energy Agency, Community Energy: Broadening The Ownership Of Renewables, IRENA Coalition for Action (2018).

2. Adam Hirsch, Yael Parag, Jos ep Guerrero, Microgrids: A Review Of Technologies, Key Drivers, And Outstanding Issues, Renewable and Sustainable Energy Reviews, Vol. 90 (2018).

3. Rehman Zafar et al, Prosumer based energy management and sharing in smart grid, Renewable and Sustainable Energy Reviews, Vol. 82, Part 1 (2018).

4. Stephen Makonin et al., AMPds: A Public Dataset for Load Disaggregation and Eco-Feedback Research, 2013 IEEE Electrical Power \& Energy Conference(2013).

5. Kaustav Basu, et al., A Comparative Study Of Low Sampling Non Intrusive Load DisAggregation, 42nd Annual Conference of IEEE Industrial Electronics Society (2016).

6. Stork M., Median filters theory and applications, Third international conference on electrical and electronics engineering papers-chamber of electrical engineering (2003).

7. Yongkui Sun, Wen Tan, Tongwen Chen, A method to remove chattering alarms using median filters, ISA Transactions, Vol.73 (2018).

8. Doron Shmilovitz, Characteristics Of Modern Nonlinear Loads And Their Influence On Systems With Distributed Generation, Int. J. Energy Technology and Policy, Vol. 5, No. 2 (2007) 
9. Abdullah Bokhari et al., Experimental Determination of the ZIP Coefficients for Modern Residential, Commercial, and Industrial Loads, IEEE Transactions On Power Delivery, Vol. 29, No. 3 (2014).

10. Adalgiza del Pilar Rios, Kodjo Agbossou, Alben Cardenas, Taylor series approximation of ZIP model for on-line estimation of residential loads' parameters, 2017 IEEE International Conference on Industrial Technology (ICIT) (2017). 